\title{
JOURNAL.RU
}

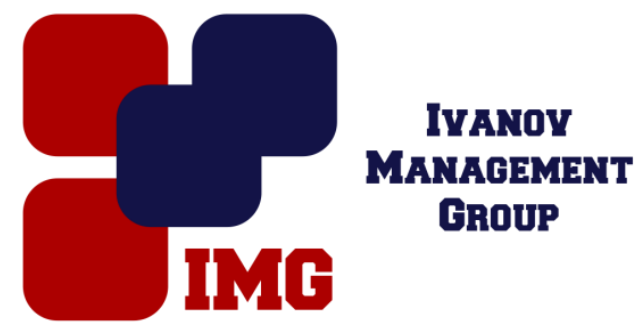

Кокадеева А.А. Саратовский соииально-экономический институт (филиал) РЭУ им. Г.В. Плеханова

Саратов, Россия

doi: 10.18411/1j-30-06-2017-01

idsp 000001:1j-30-06-2017-01

\section{Диаграммы Эйлера в решении экономических задач}

\section{Аннотация}

В данной работе будут рассмотрены возможности применения диаграмм Эйлера в решении экономических задач. На первом этапе рассматриваются основные понятия, которые связаны с диаграммами Эйлера-Венна; далее рассматривается метод решения задачи с применением диаграмм (кругов) Эйлера; на заключительном этапе на основании проделанных операций сделаны выводы о применении данного метода решения экономических задач.

Ключевые слова: диаграммы Эйлера; множество; единицы сырья

Как известно, Леонард Эйлер (1707-1783) впервые использовал круги, отображающие множества, чтобы наглядно увидеть решение поставленной задачи. Бурное развитие теории множеств и логики привело к распространению этих методов в различных областях знаний, а логически стройный и понятный аппарат постепенно вовлекается в решение различных задач экономики на различном уровне от простых повседневных задач бизнесмена до задач стратегического планирования. Необходимость учета большого количества множественных факторов влияющих на принятие решений привела к развитию современного математического аппарата, используемого для решения насущных задач. Использование диаграмм Эйлера в решении таких сложных экономических задач с одной стороны придаёт им простоту и наглядное представление решения, а с другой дает возможность при принятии решения учесть наибольшее количество ключевых факторов. Благодаря применению этого способа задачи решаются намного быстрее, а само решение становится 
наиболее понятным. Сам Эйлер, являющийся одним из известнейших математиков, писал о своих диаграммах: «Они очень подходят для того, чтобы облегчить наши размышления».

В данной работе приводятся различные примеры, иллюстрирующие возможности применения диаграмм Эйлера в исследовании разноплановых экономических задач от производственных задач до задач стратегического планирования.

Диаграммами Эйлера-Венна называются геометрические схемы, с помощью которых можно изображать множества и операции над ними. Также диаграммы Эйлера называют кругами Эйлера. С точки зрения математики под множеством понимается совокупность каких-либо объектов, выделенная по общему для них признаку. Например, множество всех рабочих данного предприятия или множество мелких фирм, производящих одинаковую продукцию. Для решения задач Леонард Эйлер использовал идею изображения множеств с помощью кругов. Здесь следует оговориться, что множества на диаграммах Эйлера-Венна могут изображаться не только кругами, но и другими фигурами, подмножества изображаются вложенными кругами или фигурами, а непересекающиеся множества изображаются непересекающимися кругами или фигурами.

Например, если объектом исследования становятся три фирмы, использующие сырьё для выработки изделий. Причём некоторые фирмы объединяются и пользуются некоторым количеством сырья совместно и известно, что 1 единицы сырья используются всеми тремя фирмами. Первая и вторая фирмы используют совместно 4 единицы сырья, вторая и третья - 5 единиц сырья, первая и третья -8 единиц сырья. Если задаться вопросом о том сколько единиц сырья в личном пользовании имеет первая фирма, если всего в её пользовании 17 единиц сырья, то ответ на данный вопрос удобнее получить с помощью диаграмм Венна-Эйлера. Кругами обозначим сырьё, используемое каждой фирмой из первых четырёх условий (рис.1).

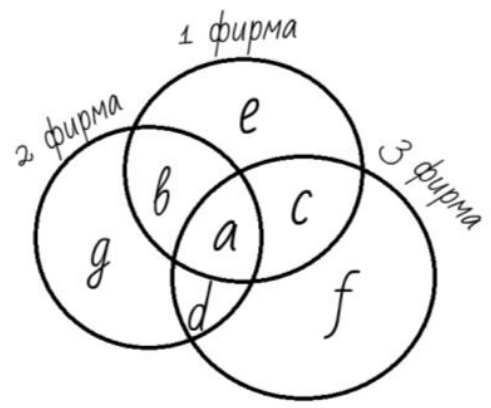

Рис.1. Диаграмма распределения сырья между тремя фирмами 
Как видно из диаграммы, представленной на рис.1 и согласно условиям поставленной задачи получаем следующие соотношения:

$$
\begin{aligned}
& a=1 \\
& a+c=4 \rightarrow c=3 \\
& a+d=5 \rightarrow d=4 \\
& a+b=8 \rightarrow b=7
\end{aligned}
$$

Из последнего условия получаем $\mathrm{a}+\mathrm{b}+\mathrm{c}+\mathrm{e}=17$,

$\mathrm{a}+\mathrm{b}+\mathrm{c}=1+7+3=11 \Rightarrow \mathrm{e}=17-11=6$

Таким образом, получаем наглядный ответ на поставленный вопрос: в личном пользовании первая фирма имеет шесть единиц сырья.

Другим ярким примером использования диаграмм Эйлера-Венна является задача определения количества изделий обработанных на конкретном оборудовании или вообще не обработанных. Например, среди ста изделий сорок штуки прошли обработку на первом станке, на втором - тридцать штук, а на третьем - двадцать восемь. Причем на первом и втором станках обработано пять изделий, на первом и третьем - десять изделий, на втором и третьем - восемь изделий, на всех трех станках обработано три изделия. Сколько изделий обработано только на первом станке и сколько изделий не обработано ни на одном из станков? Для ответа на поставленный вопрос воспользуемся диаграммами Эйлера-Венна. В качестве универсального множества выберем множество U, так как общее количество изделий известно, то $\mathrm{n}(\mathrm{U})=1000$. Пусть А - множество изделий, обработанных на первом станке, т.е. $\mathrm{n}(\mathrm{A})=420$, B - на втором станке, т.е. $\mathrm{n}(\mathrm{B})=300, \mathrm{C}$ - на третьем станке, т.е. $\mathrm{n}(\mathrm{B})=300$. Данные множества представим на диаграмме Эйлера-Венна.
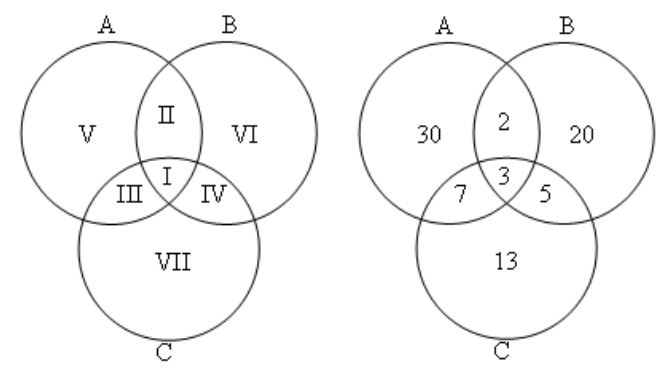

Рис.2. Диаграмма Эйлера-Венна для определения количества изделий обработанных на конкретном станке Дополнением к множеству изделий, прошедших обработку на станках, является множество необработанных деталей: $U \backslash(A \cup B \cup C)=\overline{A \cup B \cup C}$

$$
\begin{aligned}
& U\}(A \cup B \cup C)=\overline{A \cup B \cup C} n(\overline{A \cup B \cup C})=100-80=20 \\
& n(\overline{A \cup B \cup C})=100-80=20 .
\end{aligned}
$$


Таким образом, только на первом станке обработано 30 деталей, а 20 деталей не обработано ни на одном из станков.

Среди задач, к которым могут применяться диаграммы Эйлера-Венна являются задачи стратегического планирования в экономике, а так же для совершенствования планирования и прогноза политики субъекта экономики в современных условиях. Приведем пример применения диаграммы Эйлера-Венна применяются при решении задач вывода логических следствий из посылок, при решении задач прогнозирования инновации можно представить в виде диаграммы Эйлера-Венна, а так же для решения задачи о повышении эффективности бюджетных расходов характерных для субъектов Российской Федерации [Ошибка! Источник ссылки не найден.] и модернизации налогового администрирования. Например, при модернизации налогового администрирования необходимо выявлять ключевые факторы влияющие на качество прогноза.

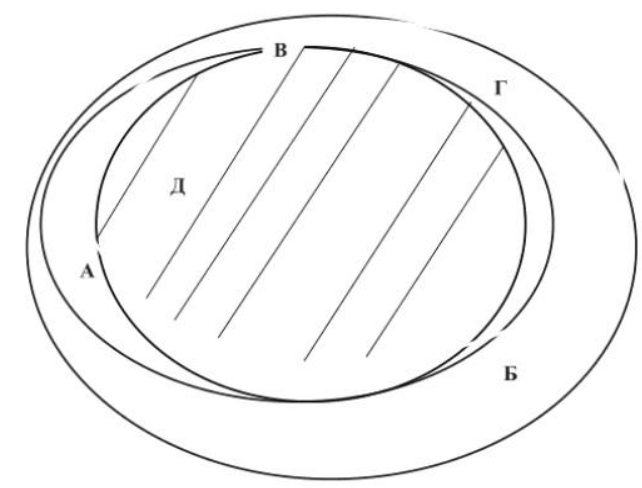

Рис.3. Использование диаграмма Эйлера-Венна для оценки качества прогноза бюджетных расходов: А-сумма налоговых поступлений, Б - сумма ожидаемой мобилизации налоговых доходов по оптимистическому сценарию; $B$ сумма ожидаемой мобилизации налоговых доходов по пессимистическому сиенарию; Г- фактическое исполнение плана налоговых поступлений

В заключения следует отметить, что использование диаграмм ЭйлераВенна в экономических задачах имеет достаточно широкое применение, а экономические выводы и результаты, полученные на их основе, дают возможность проводить исследование различных влияющих факторов на исследуемые экономические объекты и позволяю наглядно увидеть решение. Несмотря на то, что Леонард Эйлер жил более двухсот лет назад, его подход графического представления исследуемых взаимодействий множеств остается не только актуальным, но и продолжает развиваться в настоящее время. 


\section{Литература}

1. Словарь по логике. - М.: Туманит, изд. центр ВЛАДОС./ А.А.Ивин, А. Л.Никифоров. 1997.

2. Ивин А.А. Логика для журналистов. Учебное пособие/ А.А. Ивин. - М.: Аспект Пресс, 2002. - 224 с.

3. Кузичев А. С. Диаграммы Венна. История и применения. - М.: Наука, 1968. — 249 c.

4. Никифоров А.Л. Общедоступная и увлекательная книга по логике. - М.: Гнозис, 1996. - $240 \mathrm{c}$

5. Родионова Л.Н., Руднева Ю.Р. Принятие решений в инновационном менеджменте: Учебное пособие. - Уфа: Изд-во УГНТУ, 2003. - 106 с. 